\title{
QMC Calculations of Total Energy and Bond Length of Some Polyatomic Organic Molecules
}

\author{
Sylvester A. Ekong ${ }^{1 *}$ and David A. Oyegoke ${ }^{2}$ \\ ${ }^{1}$ Department of Physics \& Energy Studies, Achievers University, P.M.B. 1030 Owo, Ondo State, \\ Nigeria. \\ ${ }^{2}$ Department of Chemical Sciences, Achievers University, P.M.B. 1030 Owo, Ondo State, Nigeria. \\ *Email address: ani_sly@yahoo.co.uk
}

Keywords: QMC, DMC, VMC, Casino-code, Polyatomic molecules.

\begin{abstract}
This paper aims at determining the total energy and bond lengths of some polyatomic organic molecules, using quantum Monte Carlo (QMC) CASINO-code. The QMC code employed the VMC and DMC methods in the computations with emphasis on DMC, and using Slater-Jastrow trial wave-function formed from Hartree-Fock orbitals. The calculated results show that our reported values are in good agreement with the experimental values of both Hehre et al., and Linus Pauling. The total energies obtained in this study are 6 significant figures more accurate than those of previous studies.
\end{abstract}

\section{INTRODUCTION}

Application of quantum mechanics for the investigation of ground-state energies for molecular systems constitutes a reliable problem of interest in theoretical condensed matter physics and chemistry. Quantum-Monte-Carlo (QMC) methods have proven most suitable for obtaining precise results $[1,2]$ for the ground-state or low-lying states of molecules or atoms and are superior to perturbative methods [3]. Mohallem et al. [4] in 1986 developed a Generator Coordinate HartreeFock (GCHF) method and it was used by [4] and in 2001 by [5] to generate atomic and molecular wave-functions. In 1999 Jorge and Castro [6], developed an improved GCHF (IGCHF) which generated accurate Gaussian basis sets (GBSs) and was in 2004 used by Barreto et al. [7] to generate atomic and some diatomic molecular systems. Again, the contracted GBSs (CGBSs) generated first in 1999 by [8] and later in 2001 by [9] are enriched with polarization functions; and recently, it has been used by José and Jorge [10] to calculate, at the HF and MP2 (Mфller-Plesset second-order perturbation theory) levels, the ground state total energies of polyatomic molecules. However, the results of [10] are not in total agreement with the results of [11] reported earlier on the ground state total energies of polyatomic molecules from an ab initio molecular orbital theory (6-31G* basis sets).

Polyatomic molecules are electrically neutral groups of two or more atoms held together by covalent bonds. The molecules are distinguished from ions by their lack of electrical charge. Polyatomic molecules are of primordial importance for atmospheric applications. There are much greater difficulty in finding and fitting an adequate intermolecular potential for polyatomic molecules, especially when they are polar [12]. In addition, large molecules are likely to be deformed during collision, so that first-order perturbation theory is unsatisfactory; and it is in any case unreliable where high transfer probabilities are involved [12].

We present in this paper, quantum Monte Carlo CASINO-code, for the simulation and calculation of the ground-state total energies of some polyatomic organic molecules from two QMC methods: variational Monte Carlo (VMC) and diffusion Monte Carlo (DMC) methods. However, the most widely used QMC methods in Physics and Chemistry is the DMC method; and for detailed explanation of the QMC methods see ref: $[2,13,14]$. The reason for the significant difference in the works of the other authors mentioned above (see Table 1) could be attributed to the fact that they used variational techniques which goes a long way to limiting the accuracy of the ground-state energy due to the necessity of guessing the trial wave-function. On the basis of this, we have 
tackled the problem here by using single determinant Slater-Jastrow trial wave-function as a sampling function. In fact, reported studies have shown that trial wave functions based on single Slater configuration can reproduce experimental values with very high accuracy [15]. In addition, the ground-state total energy appears highly sensitive to the level of correlation used, especially for problems which require multi-reference treatment. Hence, it is therefore crucial to explore different types of methods to understand the impact of many-body effects more thoroughly. QMC is in principle exact; and in practice it captures the correlations at a level of $90-95 \%[13,15]$. This is something that is quite difficult to achieve by correlated methods based on expansions in basis sets. The QMC allows a direct representation of many-body effects in the wave function, at the cost of statistical uncertainty that can be reduced with more simulation time; and in addition, use in one way or the other Monte Carlo methods to handle the many-dimensional integrals that arise. Also in this study, we employ the DMC technique in calculating the bond lengths of the polyatomic organic molecules, and our results are presented in Table 1 and Table 3 respectively.

The rest of this paper is structured as follows: In Section 2, the methodology is explained with the computational details. Section 3 presents the results and discussion. Finally, the paper ends with concluding remarks in Section 4.

\section{METHODOLOGY}

The calculations have been carried out using the QMC software package, CASINO-code [16]. The CASINO-code simulations were run for the total energies of the polyatomic organic molecules: $\mathrm{C}_{2} \mathrm{H}_{2}, \mathrm{C}_{2} \mathrm{H}_{4}, \mathrm{C}_{2} \mathrm{H}_{6}$ and $\mathrm{CH}_{3} \mathrm{Cl}$, by employing the restricted Hartree-Fock (RHF) technique (where each molecule is a closed-shell system with all orbitals doubly occupied) in both VMC and DMC methods. The VMC expectation values of the operators such as the Hamiltonian are calculated with an approximate many-body trial wave function, and integrals are evaluated using Monte Carlo technique. The functional form of the wave-function is chosen to contain a number of parameters whose values are obtained by stochastic optimization. A higher accuracy is achieved in DMC method by evolving the wave-functions in imaginary time so that it decays towards the ground-state. The trial wave-function consists of a multi-determinant expansion (which describes near degeneracy or static correlation), a Jastrow factor (which captures dynamic correlation) and a back flow transformation (which allows further variations in the nodal surface). Thus, one of the most important steps in our QMC calculations is to obtain suitable trial wavefunction.

The total number of particle configurations for the simulation depends on the variational QMC steps which serve as an input parameter to the DMC. The correlated wave-function is then optimized using the variance minimization method to obtain an efficient and more accurate convergence of the total energy of each molecule. Following the generation of the Slater components, the optimization of the Jastrow function containing one-body and two-body explicit correlation terms was carried out. The optimization of the Jastrow function was as important as obtaining the Slater components since inadequacy in the Jastrow can increase the locality approximation (LA) bias. In this study, all the Jastrow parameters were optimized using a varianceminimization scheme [17] in the frame work of diffusion Monte Carlo (DMC), a variant of QMC. The CASINO-code used was run on a Linux based operating system (Ubuntu environment) having a working Fortran 90 compiler.

\section{RESULTS AND DISCUSSION}

The optimized dmc (or vmc) steps generated give rise to new configurations of electrons and nuclei at each move and because of the difference in inter-particle separation, each of these configurations will be different. The orientational polarization of the molecule may also be a contributory factor to this amongst others. The correct expectation value for each of the total energies of the polyatomic organic molecules in this work is the average total-energy of thousands of these configurations. 
Figure 1 to Figure 4, show the graphs of the results of DMC run for the following polyatomic organic molecules $\left(\mathrm{C}_{2} \mathrm{H}_{2}, \mathrm{C}_{2} \mathrm{H}_{4}, \mathrm{C}_{2} \mathrm{H}_{6}\right.$ and $\left.\mathrm{CH}_{3} \mathrm{Cl}\right)$, generated from 15,000 configurations (iteration numbers). The number of equilibration steps underwent is 2000 moves, with an imaginary time-step set at 0.002 and having a target weight of 1000. The simulation took 2000 lines of equilibration data between accepted configurations. An observation from the output files is that the DMC total energies were obtained at maximum distances of 7.32192357a.u, 11.37729022a.u, 10.84123815a.u, and 9.17706657a.u for $\mathrm{C}_{2} \mathrm{H}_{2}, \mathrm{C}_{2} \mathrm{H}_{4}, \mathrm{C}_{2} \mathrm{H}_{6}$ and $\mathrm{CH}_{3} \mathrm{Cl}$ molecules from the origin, which falls within the limits of the theoretically obtained values. This indicates greater intensity of the lowest energy levels from configuration to equilibration at a small inter-particle distances. Also, we observed from the output files acceptance ratios of $99.711 \%, 99.745 \%, 99.772 \%$ and $99.772 \%$ for the organic molecules which are in perfect agreement as predicted by $[13,15]$. This indicates that the chosen time-step does not limit the number of accepted Monte Carlo move. Hence, as the simulation equilibrates the best estimate of total energy for each, initially equal to the VMC total energy decreases significantly, and thereafter approaches a constant value which is the final DMC total energy as shown in Figure 1 to Figure 4 below.

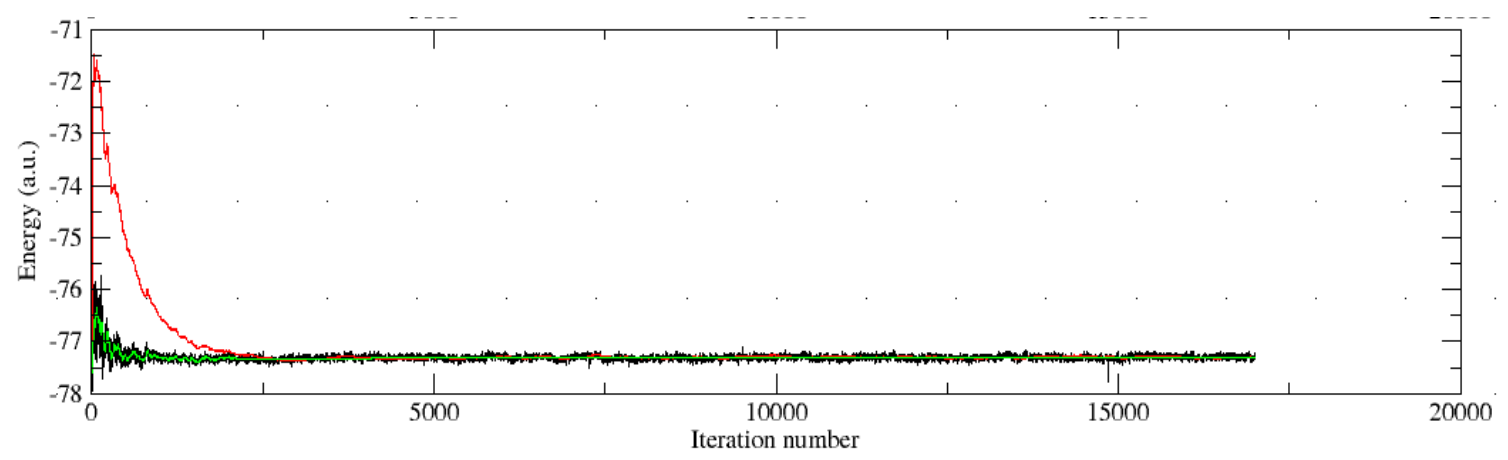

Figure 1: $\mathrm{C}_{2} \mathrm{H}_{2}$.

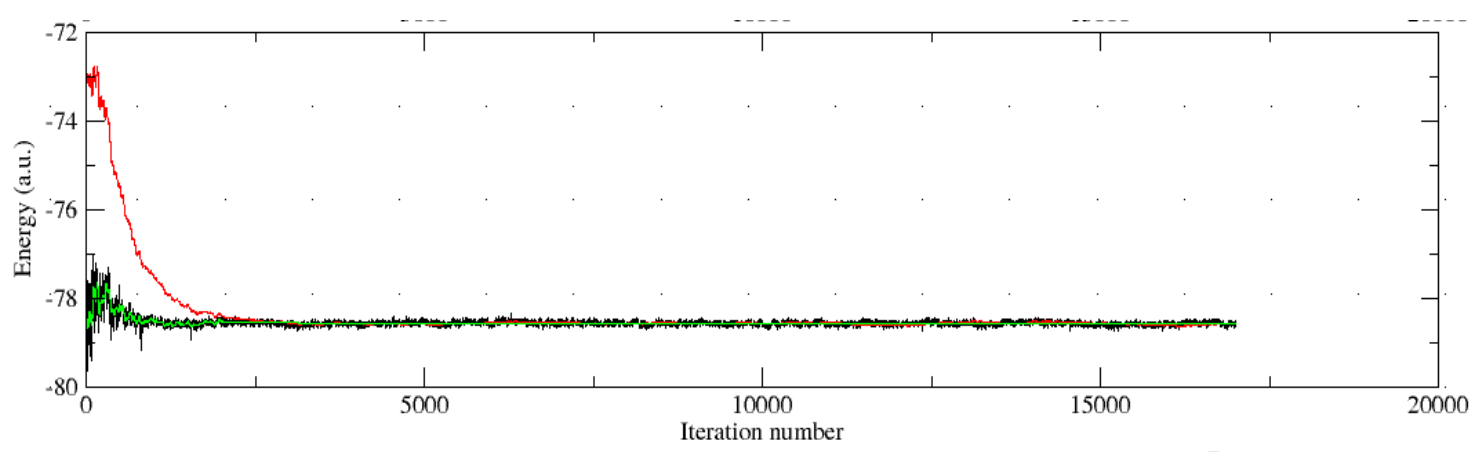

Figure 2: $\mathrm{C}_{2} \mathrm{H}_{4}$.

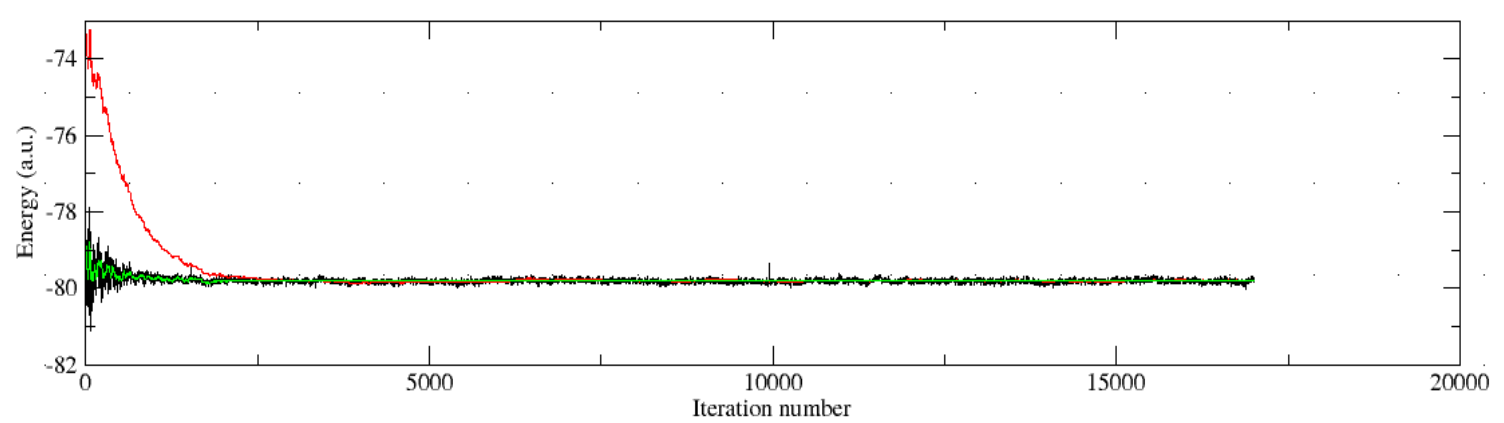

Figure 3: $\mathrm{C}_{2} \mathrm{H}_{6}$. 


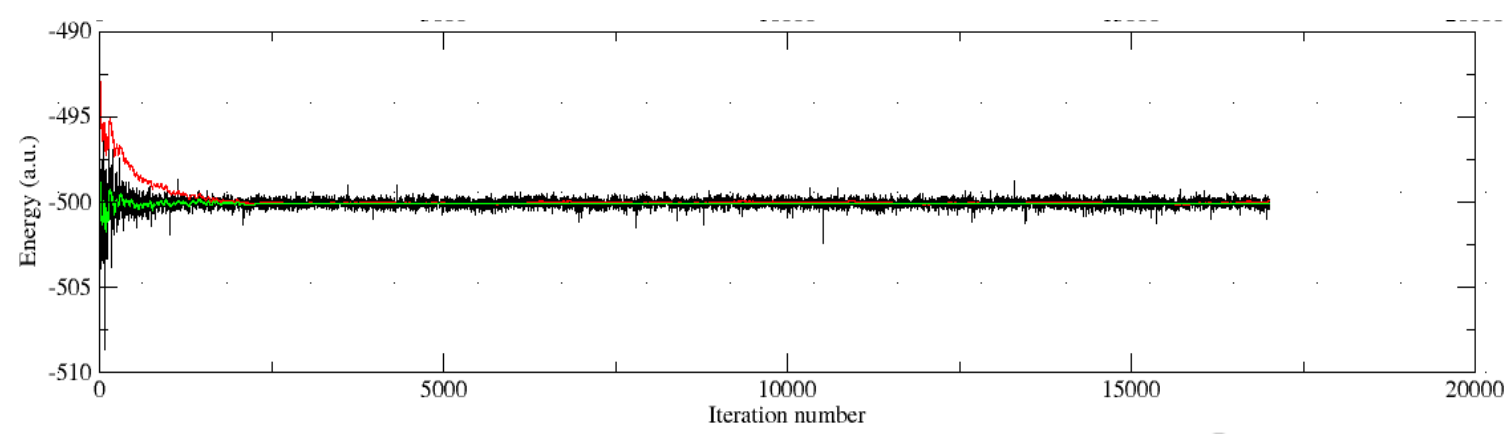

Figure 4: $\mathrm{CH}_{3} \mathrm{Cl}$.

Figures 1 to 4 shows the plot of Energies (in a.u.) as a function Iteration numbers for DMC simulations. In each graph, the noisy black line represents the local energy after each move, the green line is the current best estimate of the DMC total energy, and the red line represents the variational energy which is varied through a feedback mechanism to control the population of the configurations.

Again, from our results, it was observed that the more the DMC number of iterative steps simulated, the smaller the error bar. This means that the electronic correlation effect is higher in the DMC calculation than in the VMC. In this study, the DMC results for the polyatomic organic molecules gives a total energy values (with up to 6-decimal places) lower than those obtained by [10] as shown in Table 1. This suggests that our calculated DMC total energies may be the exacttheoretical values for the polyatomic organic molecules. The total energies and their corresponding error bars obtained from our simulations are given in Table 1 and Table 2 for both VMC and DMC methods.

Table 1: Comparative analysis of the total energy (in a.u.) of polyatomic molecules calculated by different researchers

\begin{tabular}{|l|c|c|c|c|c|}
\hline $\begin{array}{l}\text { Organic } \\
\text { Molecules }\end{array}$ & $\begin{array}{c}6-31 \mathrm{G}^{*} \\
{[10]}\end{array}$ & $\begin{array}{c}\text { FSRHGBS } \\
\text { MP2 [10] }\end{array}$ & $\begin{array}{c}\text { VMC } \\
\text { (This work) }\end{array}$ & $\begin{array}{c}\text { DMC } \\
\text { (This work) }\end{array}$ & Experiment \\
\hline $\mathrm{C}_{2} \mathrm{H}_{2}$ & -76.817107 & -76.851773 & -77.164676743656 & -77.306683328463 & - \\
\hline $\mathrm{C}_{2} \mathrm{H}_{4}$ & -78.030737 & -78.066466 & -78.373832280359 & -78.565946091036 & - \\
\hline $\mathrm{C}_{2} \mathrm{H}_{6}$ & -79.228262 & -79.263166 & -79.649567632596 & -79.806447614510 & - \\
\hline $\mathrm{CH}_{3} \mathrm{Cl}$ & - & - & -499.746916960857 & -500.080518716897 & $-502.824[20]$ \\
\hline
\end{tabular}

Table 2: Comparative analysis of the QMC error bars (in a.u.).

\begin{tabular}{|l|c|c|}
\hline $\begin{array}{l}\text { Organic } \\
\text { Molecules }\end{array}$ & $\begin{array}{c}\text { VMC } \\
\text { Error Bar }\end{array}$ & $\begin{array}{c}\text { DMC } \\
\text { Error Bar }\end{array}$ \\
\hline $\mathrm{C}_{2} \mathrm{H}_{2}$ & 0.00550454 & 0.00253571 \\
\hline $\mathrm{C}_{2} \mathrm{H}_{4}$ & 0.00547821 & 0.00323135 \\
\hline $\mathrm{C}_{2} \mathrm{H}_{6}$ & 0.00584986 & 0.00276237 \\
\hline $\mathrm{CH}_{3} \mathrm{Cl}$ & 0.02331426 & 0.00374657 \\
\hline
\end{tabular}

Similarly, the QMC CASINO-code was employed to calculate the carbon-hydrogen $(\mathrm{CH})$ bond lengths of the stated polyatomic organic molecules using the DMC technique, and the results presented in Table 3, compares favourably well with the experimental values of Hehre et al. [11]. From our results, we observed that the average deviation from experiment is $0.0463 \%$ which is highly reduced compared to the work of [10] which $0.6786 \%$ deviations away from the experimental value [11]. This result support claims that DMC provides near chemical accuracy as predicted by $[15,18]$. However, the deviation of the bond lengths in this study may be due to the single determinant Slater-Jastrow trial wave-function used, since inadequacy in the Jastrow can increase the locality approximation bias. Nevertheless, Table 3 shows a comparative analysis of the 
DMC technique with previous studies. The CASINO simulations from the DMC method shows a significant improvement in its stability towards the experimental value over the FSRHGBS method as presented in the Table 3; and this could be attributed to the stochastic nature of the DMC method.

Table 3: A comparative analysis bond lengths in $\AA$

\begin{tabular}{|l|c|c|c|}
\hline $\begin{array}{l}\text { Organic } \\
\text { Molecules }\end{array}$ & $\begin{array}{c}\text { FSRHGBS } \\
\text { MP2 [10] }\end{array}$ & $\begin{array}{c}\text { DMC } \\
\text { (This work) }\end{array}$ & Experiment \\
\hline $\mathrm{C}_{2} \mathrm{H}_{2}$ & 1.059 & 1.0659 & $1.061[11]$ \\
\hline $\mathrm{C}_{2} \mathrm{H}_{4}$ & 1.076 & 1.0848 & $1.085[11]$ \\
\hline $\mathrm{C}_{2} \mathrm{H}_{6}$ & 1.085 & 1.0928 & $1.096[11,19]$ \\
\hline $\mathrm{CH}_{3} \mathrm{Cl}$ & - & 1.7773 & $1.781[19]$ \\
\hline
\end{tabular}

\section{CONCLUSION}

With the QMC-CASINO package, the total energy and bond lengths of some polyatomic organic molecules have been calculated from both DMC and VMC methods in CASINO-code. The results obtained show that the total energy calculations from DMC gave values which are lower than the values calculated by [10], suggesting that this could be the exact total energies of the polyatomic molecules. Also, the bond length of molecules showed an average deviation of only $0.0463 \%$ from the experimental value compared to other values in literature. The results obtained show that DMC technique can give a more accurate ground-state result with very high accuracy.

\section{ACKNOWLEDGEMENTS}

We have benefited from the exchange of ideas, materials and results with other colleagues, to all of whom we offer our thanks. We thank Richard Needs, Mike Towler, Neil Drummond and Pablo López Ríos, of the Theory of Condensed Matter Group, Cavendish Laboratory, J. J. Thomson Avenue, Cambridge, UK, for the release of the CASINO-code.

\section{References}

[1] S. A. Ekong, M. T. Oloye, D. A. Oyegoke, (2015), Advances in Physics Theories and Applications, Vol. 46, pp.1-6.

[2] S. A. Ekong, O. Ebomwonyi, J. O. A. Idiodi, (2014), Journal of Nigerian Association of Mathematical Physics, Vol. 28, No.1, pp.317-326.

[3] G. Tanner, K. Richter and J.-M. Rost, (2000), Reviews of Modern Physics, Vol. 72, No. 2, pp. 505.

[4] J. R. Mohallem and M. Trsic, (1986), J. Chem. Phys. 86, pp. 5043.

[5] P. R. Librelon and F. E. Jorge, (2001), Brazil J. Phys. 31, pp. 557.

[6] F. E. Jorge and E. V. R. de Castro, (1999), Chem. Phys. Lett. 302, pp. 454.

[7] M. T. Barreto, Neto A. Canal, F. E. Jorge, (2004), Can J. Chem. 82, pp. 1237.

[9] R. Centoducatte, F. E. Jorge, E. V. R. de Castro, (2001), Int. J. Quant. Chem. 82, pp. 126.

[8] J. C. Pinheiro, F. E. Jorge, E. V. R. de Castro, (1999), J. Mol. Structure (Theochem.), 491, pp. 81.

[10] M. P. José and F. E. Jorge, (2005), Indian Journal of Chemistry, Vol. 44A, pp. 1-2.

[11] W. J. Hehre, L. Radom, P. V. R. Schleyer, J. A. Pople, (1986), Ab initio Molecular Orbital Theory (John Wiley and Sons Inc., New York. 
[12] C.H. Bamford, R.G. Compton, F.H. Tippert, (1969), The Formation and Decay of Excited Species, Comprehensive chemical kinetics, Elsevier, pp. 214.

[13] W. M. C. Foulkes, L. Mitas, R. J. Needs, and G. Rajagopal, (2001), Review of Modern Physics, Vol. 73, No. 1, Pp. 33 - 42.

[14] J. B. Anderson, (1975), J. Chem. Phys.63:1499- 1503.

[15] J. C. Grossman, (2002), Benchmark quantum Monte Carlo calculations, Journal of Chemical Physics Volume 117, Number 4, pp. 1434.

[16] R. J. Needs, M. D. Towler, N. D. Drummond, and P. López Ríos, (2012), CASINO User's Guide, version 2.11.0, Theory of Condensed Matter Group, Cavendish Laboratory, J .J. Thomson Avenue, Cambridge, UK, 2012.

[17] C. J. Umrigar, J. G. Wilson, K. W. Wilkins, (1988), Phys. Rev. Lett., 60, pp. 1719-1722.

[18] S. A. Ekong, V. A. Akpan, O. Ebomwonyi, (2015), International Letters of Chemistry, Physics and Astronomy Vol. 59 (2015) pp. 106-114.

[19] Linus Pauling, The nature of the chemical bond and the structure of molecules and crystals. An Introduction to Modern Structural Chemistry, $3^{\text {rd }}$ Edition 1960, Cornell University press, Ithaca, New York, Amazon.com, pp. 229.

[20] W. J. Grifhiths and F. M. Harris, (1988), Rapid Communications in Mass Spectrometry, Vol. 2, No 5, pp. 91. 